\title{
Stochastic Diffusion of Energetic Ions due to Lower Hybrid Waves
}

\author{
L. M. Tozawa *and L. F. Ziebell ${ }^{\dagger}$ \\ Instituto de Física \\ Universidade Federal do Rio Grande do Sul \\ Caixa Postal 15051 - CEP: 91501-970, Porto Alegre, RS, Brazil
}

Received 22 May, 1998

\begin{abstract}
This paper discusses the interaction between energetic ions and lower hybrid waves in tokamak plasmas, presenting a numerical analysis which employs a quasilinear formalism appropriated for the situation in which ion stochastic diffusion occurs. The results show that significant wave-particle interaction may take place when a population of energetic ions is present in the plasma, in partial agreement with evidence from experimental results available in the literature.
\end{abstract}

\section{Introduction}

The analysis of some experiments realized in the JET tokamak (Joint European Torus) has shown evidence of significant absorption of lower hybrid wave energy by a population of energetic ions generated by the injection of ion cyclotron radiation $[1,2]$. The description of these experimental results, obtained by direct measurements of the fast ion energy and by measurements of $\gamma$ ray and neutron emission rates, has been accompanied by theoretical analysis in which the interaction was described by a one-dimensional Fokker-Planck equation in velocity space, which includes quasilinear coefficients for ion cyclotron waves (IC) and for lower hybrid waves ( $\mathrm{LH}$ ), and a collision term accounting for the collisions with particles of the Maxwellian body of the distribution $[1,2,3]$. The diffusion coefficient for LH waves employed in the Fokker-Planck equation was intended to describe the stochastic diffusion in velocity space which may occur in the presence of sufficiently intense waves [4]. This mechanism of stochastic ion diffusion has been proposed and explained nearly two decades ago, when it was shown that the movement of an ion in a uniform magnetic field becomes stochastic in the presence of a perpendicularly propagating coher- ent electrostatic wave, if the wave amplitude exceeds a threshold [5, 4]. Due to the stochasticity, the ion diffuses in velocity space and it is possible to derive a diffusion equation to describe the time evolution of the ion distribution function, which also applies to the case of diffusion due to lower hybrid waves in the slow mode (non-vanishing $k_{\|}<<k_{\perp}$ ), in a weakly inhomogeneous magnetic field [4].

The motivation for the mentioned investigation based on the JET experiments may be summarized as follows. Lower hybrid waves are proposed as an alternative for the generation of non-inductive current in a tokamak $[6,7,8]$. With this purpose a spectrum of LH waves is launched in the tokamak, with frequency and parallel wave-number chosen in order that the waves are absorbed by electrons in the tail of the electron distribution function, by the mechanism of parallel Landau damping which occurs when the wave-particle resonance condition is satisfied. However, it had been argued that in fusion reactors the presence of energetic ions, particularly $\alpha$ particles originated from fusion reactions, would contribute to decrease the efficiency of the lower hybrid current drive, since part of the energy of the LH waves would be absorbed by the $\alpha$ particles by the mechanism of the so-called perpendicular

\footnotetext{
*E-mail: minoru@if.ufrgs.br

${ }^{\dagger} \mathrm{E}$-mail: ziebell@if.ufrgs.br
} 
Landau damping [5]. The purpose of the experiments was therefore to study, in the absence of $\alpha$ particles, the possibility of interaction between the LH waves and fast ions generated by IC waves, utilized to heat the ions in the plasma $[1,2]$. The results obtained indicate that the absorption of LH waves by fast ions indeed occurs, and therefore confirm the convenience of further investigations on this important issue concerning the dynamics of $\alpha$ particles and energetic ions in the environment of a reactor and the efficiency of the $L H$ waves for radio frequency current drive. Several examples of studies on the subject may be found in the recent literature, dedicated to the interaction of LH waves with $\alpha$ particles $[9,3,10,11,12,13]$ and other kinds of energetic ions [14, 15], including the case of interaction between LH waves and a population of energetic ions generated by neutral beam injection [16, 17, 18, 19].

In a previous publication we have addressed the subject, by considering the threshold condition for stochastic ion diffusion due to $\mathrm{LH}$ waves, originated from the analysis by Karney [4]. We have introduced models for the tokamak and the wave packet inside the tokamak, which allowed the evaluation of the wave amplitude at a given position, and its comparison with the threshold condition [20]. The results obtained show that the threshold condition may be not easily satisfied in present day large tokamaks, but can be attained in small tokamaks with relatively modest levels of wave power.

In the present paper we continue the study of stochastic diffusion of energetic ions, by means of a two-dimensional quasilinear formulation which follows the time evolution of the electron and ion distribution functions under the action of $\mathrm{LH}$ and IC waves, and collisions, in a tokamak environment approximated as a magnetized slab of plasma, as in the previous analysis [20].

The plan of the paper is the following. In Sec. II we present the models employed to describe the tokamak and the spectrum of lower hybrid waves propagating in the plasma. Sec. III is dedicated to present a short account of the quasilinear formalism utilized for the time evolution of both electron and ion distribution functions. In Sec. IV we present some numerical re- sults obtained from the application of the quasilinear formulation described in Sec. III. Finally, in Sec. V we summarize the main results of the paper, and comment on possible future developments of this investigation.

\section{Models of the tokamak and the wave packet}

Let us assume a gaussian packet of lower hybrid waves propagating in a tokamak, with spectral distribution centered at $N_{\|}=\bar{N}_{\|}$, where $N_{\|}$is the component of $\mathbf{N}=c \mathbf{k} / \omega$ parallel to the ambient magnetic field ( $\mathbf{k}$ is the wave vector, and $\omega$ is the angular wave frequency). Let us assume that the half-width of the spectrum is given by $\Delta N_{\|}$,

$$
S\left(N_{\|}\right)=S_{0} \frac{e^{-\left(N_{\|}-\bar{N}_{\|}\right)^{2} /\left(\Delta N_{\|}\right)^{2}}}{\sqrt{\pi}\left(\Delta N_{\|}\right)},
$$

where $\int d N_{\|} S\left(N_{\|}\right)=S_{0}$, the local wave intensity. The local amplitude of the electric field is related to the energy flux, and may be given by [21]

$$
E_{0}\left(N_{\|}\right)=\left(\frac{16 \pi}{\left(4 \pi \epsilon_{0}\right) c} \frac{|B|}{|\partial D / \partial \mathbf{N}|} S\left(N_{\|}\right)\right)^{1 / 2}
$$

In order to atribute to these quantities a numerical value at a given position, it is necessary to describe with more details the wave propagation and the energy deposition of the wave. However, details about these processes are difficult to estimate, since lower hybrid waves suffers considerable refraction inside the plasma. Therefore, we will assume a simplifying model, which will be useful to provide an estimate about the magnitude of the quasilinear effect on the plasma electrons compared to the effect on the ion distribution, and therefore provide an estimate about the feasibility of the stochastic diffusion, as a function of wave and plasma parameters.

For these purposes a simplified tokamak model described by a slab of plasma shall be satisfactory, with the following profiles

$$
\begin{aligned}
& n_{\alpha}(x)=n_{\alpha}(0)\left(1-\frac{x^{2}}{a^{2}}\right) \\
& T_{\alpha}(x)=T_{\alpha}(0)\left(1-\frac{x^{2}}{a^{2}}\right)^{2} \\
& B_{0}(x)=B_{0}(0)\left(1+\frac{x}{R}\right)^{-1},
\end{aligned}
$$


where $a$ is the minor radius, $R$ is the major radius, and $x$ is the radial coordinate in the equatorial plane, with the origin in the center of the plasma column. $n_{\alpha}(0)$, $T_{\alpha}(0)$, and $B_{0}(0)$ are respectively the density and electron temperature of species $\alpha$ and the ambient toroidal magnetic field, at the center of the plasma.

The half-width along the $z$-axis occupied by the wave packet propagating with spectral half-width $\Delta N_{\|}$may be approximately estimated as $\Delta z=$ $2 c /\left(\omega\left(\Delta N_{\|}\right)\right)$. If we assume a poloidal width $\Delta \theta$ for the wave packet, the area affected by the wave in a magnetic surface of radius $r$ will be given by $S_{\omega}=2(\Delta z)(\Delta \theta) r$, while the area of the magnetic surface itself will be $S=4 \pi^{2} r R$, and therefore the fraction of a given magnetic surface which is affected by the wave may be estimated as

$$
\gamma \equiv \frac{S_{\omega}}{S}=\frac{\Delta z \Delta \theta}{2 \pi^{2} R}
$$

This quantity can also be regarded as an estimation of the fraction of time in which a particle moving on a magnetic surface at radius $r$ remains under the influence of the wave.

After these geometric considerations, it is possible to evaluate the local wave intensity as the ratio between the wave power, $W_{0}$, and the surface occupied by the wave front, estimated as $S_{\omega}$, resulting that $S_{0}=W_{0} / S_{\omega}$. This rather rough estimate of wave intensity tends to very large intensities near the center of the tokamak, and indeed diverges for $r \rightarrow 0$, since $S_{\omega} \rightarrow 0$. In order to avoid this divergence, we arbitrarily set a minimum value for $S_{\omega}$ and a corresponding maximum value for $S_{0}$, chosen as the values of $S_{\omega}$ and $S_{0}$ at $r=2$ $\mathrm{cm}$. Even with this cut-off value which avoid the divergence of the wave intensity at $r \rightarrow 0$, it is possible that this simplified model is super-estimating the local magnitude of the wave electric field, for the central regions. If that is the case, it will at least be useful to provide limits to the possibility of stochastic diffusion, as a function of wave energy.

For application of this scheme, the relevant parameters for the lower hybrid waves will be $\omega, \bar{N}_{\|}, \Delta N_{\|}$, and $W_{0}$. Another necessary parameter is $\Delta \theta$, which will be assumed fixed for all the ensuing cases, as $\Delta \theta=\pi / 4$ [4]. For given values of $\omega$ and $N_{\|}$, the dispersion relation for LH waves must be solved in order to obtain $N_{\perp}$.

\section{Quasilinear formulation for electron and ion distributions}

From basic quasilinear theory, it is known that the time evolution of the distribution function for particles of population $\alpha$, at position $x$, under the action of lower hybrid and ion cyclotron waves and a collision term, may be described by an equation of the following form

$$
\partial_{\tau_{\alpha}} f_{\alpha}=\left(\partial_{\tau_{\alpha}} f_{\alpha}\right)_{l h}+\left(\partial_{\tau_{\alpha}} f_{\alpha}\right)_{i c}+\left(\partial_{\tau_{\alpha}} f_{\alpha}\right)_{c o l}
$$

where $f_{\alpha}=f_{\alpha}\left(u_{\|}, u_{\perp}, \tau_{\alpha}, x\right) . \quad \tau_{\alpha}$ is the time normalized to the collision time, defined as the inverse of the local collision frequency, $\nu_{\alpha}(x)=$ $\Lambda(x) \omega_{p \alpha}^{4}(x) / 8 \pi n_{\alpha}(x) v_{\alpha}^{3}(x) . \quad \Lambda(x)$ is the Coulomb logarithm at position $x, \omega_{p \alpha}(x)$ is the angular plasma frequency at the same position, $n_{\alpha}(x)$ is the local density of particles of population $\alpha$, and $v_{\alpha}(x)=$ $\left(T_{\alpha}(x) / m_{\alpha}\right)^{1 / 2}$ is the thermal velocity for these particles. The $u_{\perp}$ and $u_{\|}$quantities that appear as arguments of $f_{\alpha}$ are the components of the velocity in the directions respectively perpendicular and parallel to the ambient magnetic field, normalized to $v_{\alpha}$. The symbol $\alpha$ is not appended to the components of $u$ in order to avoid excessive burden to the notation. The first term of the right-hand side of Eq. (5) indicates the action of the lower hybrid waves, the second term represents the effect of ion cyclotron waves, and the last term expresses the effect of the collisions. In what follows, explicit expressions for these terms will be given, adequated for each particle species.

\section{A. Quasilinear formalism for the electron distri- bution function}

For the case of the electron distribution function, the first term in the right hand side of equation (5) may be obtained from conventional quasilinear formalism,

$$
\left(\partial_{\tau_{e}} f_{e}\right)_{l h}=\partial_{u_{\| l}}\left(d_{l h} \partial_{u_{\|}} f_{e}\right),
$$

where $u$ is defined as $v / v_{e}$, and where $d_{l h}$ is the lower hybrid diffusion coefficient, which in the case of a spectrum of LH waves as we have introduced may take the following form 


$$
d_{l h}\left(u_{\|}\right)=\frac{16 \pi^{2}}{m_{e} \omega_{p e}^{2} \omega \Lambda\left|u_{\|}\right|} \gamma\left(\frac{|B|}{|A|} \frac{\left.\left.\left|D_{11} D_{22}-\right| D_{12}\right|^{2}\right|^{2}}{|\partial D / \partial \mathrm{N}|} S\left(N_{\|}\right)\right)_{N_{\|}=\mu_{e}^{1 / 2} / u_{\|}}
$$

In the above expression $\omega$ is the frequency of lower hybrid waves, $\mu_{e} \equiv m_{e} c^{2} / T_{e}$, and $S\left(N_{\|}\right)$is the spectrum of energy flux for the lower hybrid waves. This form of the diffusion coefficient is based in a well known formulation found in the literature [21], but here we have written explicitly the factor $\gamma$, defined by Eq. (4), accordingly with the procedure to be adopted also for the diffusion coefficient acting on the ion distribution. The $D_{i j}$ are defined by $D_{i j}=N_{i} N_{j}-N^{2} \delta_{i j}+\epsilon_{i j}$, where the $N_{i}$ are the components of $\mathbf{N}$, and the $\epsilon_{i j}$ are the components of the cold plasma dielectric tensor. The quantities $A$ and $B$ are given by the following

$$
\begin{gathered}
A=\left|D_{13} D_{22}-D_{12} D_{23}\right|^{2}+\left|D_{23} D_{11}-D_{12}^{*} D_{13}\right|^{2}+\left.\left.\left|D_{11} D_{22}-\right| D_{12}\right|^{2}\right|^{2}, \\
B=D_{11} D_{22}-\left|D_{12}\right|^{2}+D_{11} D_{33}-\left|D_{13}\right|^{2}+D_{22} D_{33}-\left|D_{23}\right|^{2} .
\end{gathered}
$$

while $D$ is the dispersion relation for the LH waves, obtained from the determinant of the $D_{i j}$,

$$
D=\epsilon_{11} N_{\perp}^{4}+N_{\perp}^{2}\left[\left(\epsilon_{11}+\epsilon_{33}\right)\left(N_{\|}^{2}-\epsilon_{11}\right)-\epsilon_{12}^{2}\right]+\epsilon_{33}\left[\left(\epsilon_{11}-N_{\|}^{2}\right)^{2}+\epsilon_{12}^{2}\right]=0
$$

The second term in Eq. (5) vanishes for the case of $\alpha=e$, since there is no effect from the ion cyclotron waves on the time evolution of the electron distribution function.

For the third term, which is the collision term, we use a simplified form describing the interaction of electrons belonging to the electronic tail with electrons and ions from the body of their respective distribution functions, since the velocity of the tail electrons is much larger than both ion and electron thermal velocities [21]. This term is given by

$$
\left(\partial_{\tau_{e}} f_{e}\right)_{c o l}=\frac{Z^{2}+1}{u^{3} \sin \theta} \partial_{\theta}\left(\sin \theta \partial_{\theta} f_{e}\right)+\frac{2}{u^{2}} \partial_{u}\left(\frac{1}{u} \partial_{u} f_{e}+f_{e}\right),
$$

where $\theta$ is the angle between the particle momentum and the ambient magnetic field and $Z$ is the effective ion charge.

\section{B. Quasilinear formalism for the ion distribution function}

For the quasilinear evolution of the ion distribution function, an additional assumption will be made. We are using a gaussian spectrum of $\mathrm{LH}$ waves, but if it is narrow we may assume that only the central ray is significant for the determination of the diffusion coefficient which will be used in Eq. (5). That is, we will consider the case of a single monochromatic LH wave acting on the ions. We intend to investigate the role of this simplifying assumption in the near future, but it is useful in the present case because it may provide a limiting case for the study of the quasilinear effects of the LH ions on energetic ions.

Therefore, we follow the formalism developed by Karney [4]. According to this formalism, the diffusion in velocity space produced by a lower hybrid wave with angular frequency $\omega$ and components of the wave number $N_{\perp}$ and $N_{\|}$may be described by the following equation, in normalized form,

$$
\left(\partial_{\tau_{i}} f_{i}\right)_{l h}=\frac{1}{u_{\perp}} \partial_{u_{\perp}}\left(u_{\perp} D_{l h} \partial_{u_{\perp}} f_{i}\right)
$$

where the ion thermal velocity is used for velocity normalization $\left(u \equiv v / v_{i}\right)$, and where 


$$
D_{l h}\left(u_{\perp}\right)=\left\{\begin{array}{l}
\frac{\pi}{4} \frac{E_{0}^{2}}{c^{2} B_{0}^{2}} \frac{\mu_{i}^{2}}{N_{\perp}^{2} u_{\perp}^{2}} \frac{\Omega_{i}}{\nu_{i}}\left|H_{\nu}^{(1) \prime}(\nu)\right|^{2} g^{2}(\mathcal{A}) \gamma, \text { for } u_{\perp} \geq u_{p h}, \\
D_{l h}\left(u_{p h}\right), \text { for } u_{p h}-u_{t r} \leq u_{\perp}<u_{p h} \\
D_{l h}\left(u_{p h}\right)\left[u_{\perp}-\left(u_{p h}-2 u_{t r}\right)\right]^{2}, \text { for } u_{p h}-2 u_{t r} \leq u_{\perp}<u_{p h}-u_{t r} \\
0, \text { for } u_{\perp}<u_{p h}-2 u_{t r} .
\end{array}\right.
$$

Moreover,

$$
\begin{gathered}
u_{p h}=\omega /\left(k_{\perp} v_{i}\right), \quad u_{t r}=\left(q_{i} E_{0} / m_{i} k_{\perp}\right)^{1 / 2} / v_{i}, \\
\mathcal{A}=\frac{\alpha \nu}{r}\left|H_{\nu}^{(1) \prime}(\nu)\right|,
\end{gathered}
$$

with the introduction of the following definitions

$$
\nu=\omega / \Omega_{i}, \quad r=k_{\perp} v_{\perp} / \Omega_{i},
$$

and

$$
\alpha=\frac{E_{0}}{B_{0}} \frac{k_{\perp}}{\Omega_{i}}=\frac{E_{0}}{B_{0}} \frac{N_{\perp} \nu}{c},
$$

where $N_{\perp}$ is the perpendicular component of $\mathbf{N}, \Omega_{i}$ is the ion cyclotron frequency, $E_{0}$ is the amplitude of the wave electric field, and $B_{0}$ is the ambient magnetic field. We have also defined $\mu_{i}=m_{i} c^{2} / T_{i}$. The quantities $H_{\nu}^{(1)}$ and $H_{\nu}^{(1) \prime}$ are respectively the Hankel function of first kind and its derivative, given by

$$
\begin{aligned}
\left|H_{\nu}^{(1)}(r)\right| & \simeq(2 / \pi)^{1 / 2}\left(r^{2}-\nu^{2}\right)^{-1 / 4}, \\
\left|H_{\nu}^{(1) \prime}(r)\right| & \simeq(2 / \pi)^{1 / 2}\left(r^{2}-\nu^{2}\right)^{1 / 4} / r,
\end{aligned}
$$

for $r \geq \nu+(\nu / 2)^{1 / 3}[4]$; for $r<\nu+(\nu / 2)^{1 / 3}$, it is used

$$
\begin{aligned}
\left|H_{\nu}^{(1)}(r)\right| \simeq\left|H_{\nu}^{(1)}\left(\nu+(\nu / 2)^{1 / 3}\right)\right|, \\
\left|H_{\nu}^{(1) \prime}(r)\right| \simeq\left|H_{\nu}^{(1) \prime}\left(\nu+(\nu / 2)^{1 / 3}\right)\right| .
\end{aligned}
$$

In Eq. (11) the threshold condition is expressed by the quantity $g(\mathcal{A})$. This quantity is zero for $\mathcal{A}$ smaller than $\mathcal{A}_{s}$ and grows fast to $g(\mathcal{A})=1$ for $\mathcal{A}$ larger than $\mathcal{A}_{s}[4]$,

$$
g(\mathcal{A})=\max \left[1-\mathcal{A}_{s}^{2} / \mathcal{A}^{2}, 0\right] .
$$

The stochasticity condition defines the situation in which a stochastic layer occupies a substantial fraction of the phase space, and therefore the diffusion becomes effective. This condition does not allow a precise definition of $\mathcal{A}_{s}$. Karney sets the requirement that for $\mathcal{A}=\mathcal{A}_{s}$ the phase change suffered by a particle in the wave-particle resonance would be equal to $\pi / 2$, and therefore assumes that $\mathcal{A}_{s}=0.25$ [4]. The same condition is assumed in the present work.

Another quantity appearing in Eq. (11) is the fraction of time in which the particles remain under the effect of the lower hybrid waves, denoted by $\gamma$, which is a quantity dependent on the existence of models for the tokamak and the wave packet. According to the models which we have introduced, this fraction of time is given by Eq. (4).

For the second term appearing in Eq. (5), we use a simplified form, which guarantees the creation of a population of energetic ions with large perpendicular energy. The existence of this population is essential for our present purpose of analysis of their interaction with LH waves, but we will not be concerned here with the details of the interaction between the ions and the IC waves. The model diffusion term to be utilized for IC waves is the following [2, 22]

$$
\left(\partial_{\tau_{i}} f_{i}\right)_{i c}=\frac{1}{u_{\perp}} \partial_{u_{\perp}}\left(u_{\perp} D_{i c} \partial_{u_{\perp}} f_{i}\right),
$$

where we assume

$$
D_{i c}=D_{i c 0} \frac{e^{-\left(u-\bar{u}_{i c}\right)^{2} /(\Delta u)^{2}}}{\Delta u \sqrt{\pi}} .
$$

It is easy to see that $\bar{u}_{i c}$ indicates the velocity of maximum value of the diffusion coefficient and $\Delta u$ is the half-width of the spectrum, with $D_{i c 0} \equiv \int d u D_{i c}(u)$.

For the collision term, we consider that the energetic ions constitute a tenuous population and mostly collide 
with the ions of the Maxwellian body of the distribution, as well as with Maxwellian electrons. Therefore, it is possible to neglect the nonlinear effect due to collisions within the energetic tail. We start from expres- sions available in the literature for the Fokker-Planck collision term for energetic ions [23], and after straightforward but tedious algebraic manipulations arrive to the following

$$
\begin{aligned}
\left(\partial_{\tau_{i}} f_{i}\right)_{\text {coll }}= & \frac{1}{Z^{2}}\left\{\frac{m_{i}}{m_{e}} \frac{2}{u^{3}} \Psi\left(u_{e} / \sqrt{2}\right) \partial_{u}^{2} f_{i}+\frac{m_{i}}{m_{e}} \frac{2}{u^{2}}\left[\Psi\left(u_{e} / \sqrt{2}\right)+\left(\frac{m_{e}}{m_{i}}\right)^{3 / 2} \frac{u}{\sqrt{2}} \Phi^{\prime}\left(u_{e} / \sqrt{2}\right)-\frac{1}{u^{2}} \Psi\left(u_{e} / \sqrt{2}\right)\right] \partial_{u} f_{i}\right. \\
+ & {\left.\left[\Phi\left(u_{e} / \sqrt{2}\right)-\frac{m_{i}}{m_{e}} \frac{1}{u^{2}} \Psi\left(u_{e} / \sqrt{2}\right)\right] \frac{1}{u^{3}} \partial_{\mu}\left[\left(1-\mu^{2}\right) \partial_{\mu} f_{i}\right]+\left(\frac{m_{e}}{m_{i}}\right)^{1 / 2} \sqrt{2} \Phi^{\prime}\left(u_{e} / \sqrt{2}\right) f_{i}\right\} } \\
+ & \left\{\frac{2}{u^{3}} \Psi(u / \sqrt{2}) \partial_{u}^{2} f_{i}+\frac{2}{u^{2}}\left[\Psi(u / \sqrt{2})+\frac{u}{\sqrt{2}} \Phi^{\prime}(u / \sqrt{2})-\frac{1}{u^{2}} \Psi(u / \sqrt{2})\right] \partial_{u} f_{i}\right. \\
+ & {\left.\left[\Phi(u / \sqrt{2})-\frac{1}{u^{2}} \Psi(u / \sqrt{2})\right] \frac{1}{u^{3}} \partial_{\mu}\left[\left(1-\mu^{2}\right) \partial_{\mu} f_{i}\right]+\sqrt{2} \Phi^{\prime}(u / \sqrt{2}) f_{i}\right\} }
\end{aligned}
$$

where the terms divided by the factor $Z^{2}$ refer to ion-electron collisions, and the remaining terms to ion-ion collisions, and where $u_{e} \equiv\left(m_{e} / m_{i}\right)^{1 / 2} u$. Moreover, we have

$$
\Psi(x)=\Phi(x)-x \Phi^{\prime}(x), \quad \Phi(x)=\frac{2}{\sqrt{\pi}} \int_{0}^{x} e^{-t^{2}} d t, \quad \Phi^{\prime}(x)=\frac{2}{\sqrt{\pi}} e^{-x^{2}}
$$

\section{Numerical results of the quasilinear analysis}

The formalism described in Sec. III can be now applied for evaluation of the time evolution of the distribution function of ions and electrons.

\section{A. Implementation of the quasilinear formalism}

In order to solve numerically equation (5) for ion and electron distribution functions, we write if entirely as function of the variables $u$ and $\mu=\cos \theta$, with the $(u, \mu)$ space described by a discrete grid of $151 \times$ 31 points. The $u$ limits of the grid are $u=0$ and $u=u_{\text {lim }}=10$, and the limits in $\mu$ are $\mu= \pm 1$. It is assumed that the distribution remains Maxwellian at $u=u_{l i m}$ and beyond, and satisfy the condition $\partial f / \partial \theta=0$ at $\mu=-1$ and $\mu=1$. The set of finite difference equations obtained from the quasilinear equation is then solved numerically, using the alternating directions implicit method (ADI) [24].

For the diagnostics of the results obtained, we define a series of auxiliary quantities, the parallel distribution function, the perpendicular distribution function, the perpendicular temperature, the electronic current density, and the dimensionless power dissipated by IC and LH waves, defined respectively as follows

$$
\begin{gathered}
f_{\alpha||}\left(u_{\|}, \tau_{\alpha}, x\right)=2 \pi \int_{0}^{\infty} d u_{\perp} u_{\perp} f_{\alpha}\left(u_{\|}, u_{\perp}, \tau_{\alpha}, x\right) \\
f_{\alpha \perp}\left(u_{\perp}, \tau_{\alpha}, x\right)=\int_{-\infty}^{\infty} d u_{\|} f_{\alpha}\left(u_{\|}, u_{\perp}, \tau_{\alpha}, x\right) \\
T_{\alpha \perp}\left(u_{\|}, \tau_{\alpha}, x\right)=\frac{2 \pi T_{e}}{f_{\alpha \|}} \int_{0}^{\infty} d u_{\perp} u_{\perp} \frac{u_{\perp}^{2}}{2} f_{\alpha}\left(u_{\|}, u_{\perp}, \tau_{\alpha}, x\right),
\end{gathered}
$$




$$
\begin{gathered}
J_{\alpha}\left(\tau_{\alpha}, x\right)=-\frac{e c n_{e}}{\sqrt{\mu_{e}}} \int d^{3} u u_{\|} f_{e}\left(u_{\|}, u_{\perp}, \tau_{\alpha}, x\right) \\
\left(P_{\alpha}\right)_{i c}\left(\tau_{\alpha}, x\right)=\int d^{3} u \frac{u^{2}}{2}\left(\partial_{\tau_{\alpha}} f_{\alpha}\left(u_{\|}, u_{\perp}, \tau_{\alpha}, x\right)\right)_{i c} \\
\left(P_{\alpha}\right)_{l h}\left(\tau_{\alpha}, x\right)=\int d^{3} u \frac{u^{2}}{2}\left(\partial_{\tau_{\alpha}} f_{\alpha}\left(u_{\|}, u_{\perp}, \tau_{\alpha}, x\right)\right)_{l h} .
\end{gathered}
$$

For the evaluation of $\left(P_{\alpha}\right)_{i c}$ and $\left(P_{\alpha}\right)_{l h}$, of course, the corresponding terms in Eq. (5) are inserted in the integrand at the right-hand side.

\section{B. Results of the numerical analysis}

We consider plasma parameters representative of large tokamaks, similar to those of the JET tokamak (Joint European Torus), with profiles given by Eq. (3). The parameters are the following: $n_{e}(0)=3.5 \times 10^{19}$ $\mathrm{m}^{-3}, T_{e}(0)=5.0 \mathrm{keV}$, equal ion and electron densities and temperatures, and $B_{0}(0)=3.0 \mathrm{~T}$. For minor radius we use $a=1.2 \mathrm{~m}$, and for the major radius we use $R=5.0 \mathrm{~m}$, somewhat larger than the JET radius.

For the LH waves we consider parameters already utilized in our previous analysis about the threshold condition [20], namely $\omega=1.5 \omega_{l h}, \bar{N}_{\|}=2.53, \Delta N_{\|}=$ 0.2 , and three values of wave power, $W_{0}=2.0,1.5$, and $1.0 \mathrm{MW}$. The choice of $\bar{N}_{\|}=2.53$ means that the center of the LH spectrum will be resonating with electrons at four thermal velocities, which is expected to assure good efficiency in current generation. For practical reasons, the gaussian spectra has been assumed to exist between $\bar{N}_{\|}-2 \Delta N_{\|}$and $\bar{N}_{\|}+2 \Delta N_{\|}$, vanishing outside these limits. Numerically, the LH spectrum is described by an array of 61 points. For the IC waves we assume $D_{i c 0}=0.5, \bar{u}_{i c}=5.0$, and $\Delta u=1.0$, to be used with the model coefficient given by Eq. (15).

The formalism developed effectively uncouples ion and electron distributions, since the collision term only includes collisions of the resonating particles with the Maxwellian background. Therefore, the study of the two distributions can be made independently. We use a time step $\Delta \tau_{\alpha}=1 . \times 10^{-3}$, running the code until a steady-state is nearly attained, with the wave effects on the distribution function nearly balanced by collision effects.

Let us start by the analysis of the electron distribution function, which is not affected by the IC diffusion term. We choose to display the results obtained at the central position of the tokamak, $x=0 \mathrm{~m}$. In Fig. 1 we show the quantity $-\ln f_{e \|}$, as a function of $u_{\|}\left|u_{\|}\right|$, for $W_{0}=1.0,1.5$, and $2.0 \mathrm{MW}$, at $\tau_{e}=100$. For comparison, a Maxwellian distribution appears in this representation, in the shape of the letter V. It is easily seen the assymetry caused by the LH waves toward the side of positive $u_{\|}$, although some effect is also seen in the side of negative $u_{\|}$, caused by collisional diffusion. The perpendicular distribution is also affected by collisional diffusion, as can be seen is Fig. 2, which shows $\ln f_{e \perp}$, as a function of $u_{\perp}^{2} / 2$, for $W_{0}=1.0,1.5$, and 2.0 MW, also at $\tau_{e}=100$. This excess of perpendicular energy is not equally distributed along the distribution, as demonstrated by the peaks in perpendicular temperature exhibitted in Fig. 3. These features of the electron distribution function under the effect of LH waves are well known and are shown here only for the sake of completicity [21, 25, 26].

Still regarding the dynamics of the interaction between LH waves and electrons, in Fig. 4 we show the time evolution of the power dissipated by lower hybrid waves on the electron distribution function, for the same parameters used in Fig. 1. Instead of plotting the quantity $\left(P_{e}\right)_{l h}$, as given by Eq. (17), we present in Fig. 4 the time evolution of the energy dissipated on the electrons by unit of time measured in the time scale of the ions, a quantity which we denote by $\hat{P}_{e}$,

$$
\left(\hat{P}_{e}\right)_{l h}\left(\tau_{e}, x\right) \equiv \int d^{3} u \frac{u^{2}}{2}\left(\partial_{\tau_{i}} f_{e}\left(u_{\|}, u_{\perp}, \tau_{e}, x\right)\right)_{l h}=\frac{\nu_{e}}{\nu_{i}}\left(P_{e}\right)_{l h}\left(\tau_{e}, x\right)
$$


This quantity has been defined here because it is useful for direct comparison between the energy dissipated on the electrons and the energy dissipated on the ions, measured in the same scale of time.

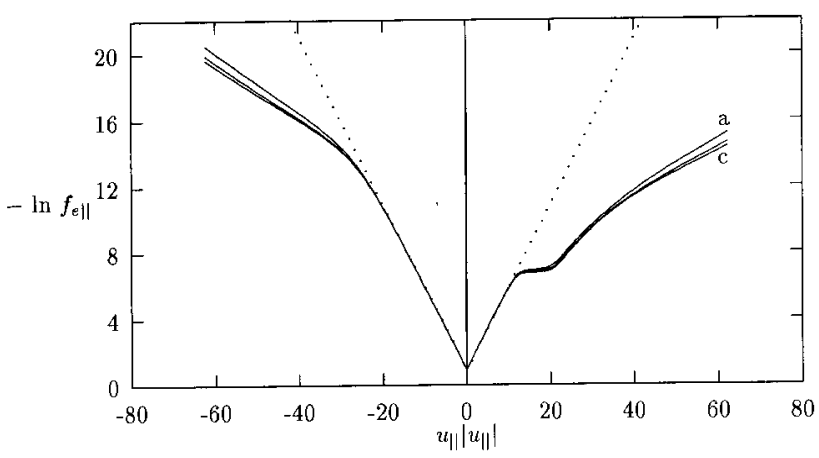

Figure 1. $-\ln f_{e \|}$, vs. $u_{\|}\left|u_{\|}\right|$, at $x=0 \mathrm{~m}$ and $\tau_{e}=100$, for $\omega=1.5 \omega_{l h}, \bar{N}_{\|}=2.53$, and $\Delta N_{\|}=0.2$. (a) $W_{0}=1.0$ $\mathrm{MW}$; (c) $W_{0}=2.0 \mathrm{MW}$; between these two, the curve for $W_{0}=1.5 \mathrm{MW}$. The dotted line represents the Maxwellian distribution. The plasma parameters are $n_{e}(0)=3.5 \times 10^{19}$ $\mathrm{m}^{-3}, T_{e}(0)=5.0 \mathrm{keV}, B_{0}(0)=3.0 \mathrm{~T}, a=1.2 \mathrm{~m}$, and $R=$ $5.0 \mathrm{~m}$.

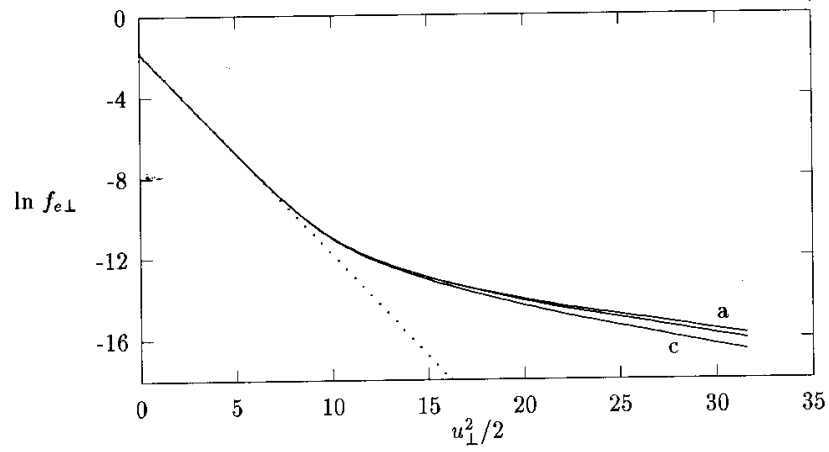

Figure 2. In $f_{e \perp}$, vs. $u_{\perp}^{2} / 2$, at $x=0 \mathrm{~m}$ and $\tau_{e}=100$, for $\omega=1.5 \omega_{l h}, \bar{N}_{\|}=2.53$, and $\Delta N_{\|}=0.2$. (a) $W_{0}=1.0$ $\mathrm{MW}$; (c) $W_{0}=2.0 \mathrm{MW}$; between these two, the curve for $W_{0}=1.5 \mathrm{MW}$. The dotted line represents the Maxwellian distribution. The plasma parameters are the same as in Fig.1.

We investigate now the time evolution of the ion distribution function. The first noticeable feature, which has been anticipated in a previous publication [20], is that for the frequency utilized the diffusion coefficient for $\mathrm{LH}$ waves vanishes for $W_{0}=1.0 \mathrm{MW}$, and is barely significant for $W_{0}=1.5 \mathrm{MW}$. For larger wave frequencies $\left(\omega / \omega_{l h} \geq 2.0\right)$, the diffusion coefficient would be null even for $W_{0}=2.0 \mathrm{MW}$ [20].

These results seem to disagree with experimental results which report absorption of LH waves by energetic ions, at frequency $\omega / \omega_{l h}=3.7$, even for wave power of 1.0 MW [1]. As discussed on our previous analysis on the threshold condition [20], one possible reason for the difference is that in the experimental situation a spectrum of waves is present, while the theory utilized assumes a monochromatic LH wave acting on the energetic ions. We intend to continue our investigation on the subject, by considering the effect of the presence of a group or spectrum of waves, but we consider that the simplicity of the slab model and narrow gaussian spectra utilized here may provide at least an useful qualitative guide to the phenomena involved.

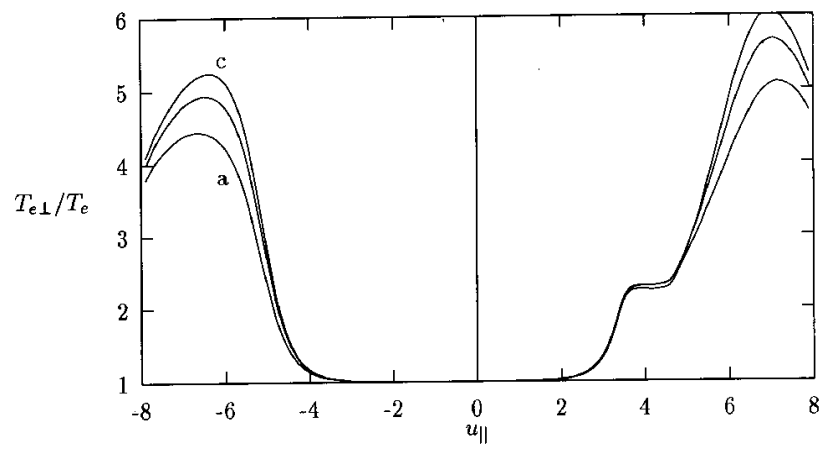

Figure 3. $T_{e \perp} / T_{e}$ vs. $u_{\|}$, at $x=0 \mathrm{~m}$ and $\tau_{e}=100$, for $\omega=1.5 \omega_{l h}, \bar{N}_{\|}=2.53$, and $\Delta N_{\|}=0.2$. (a) $W_{0}=1.0$ $\mathrm{MW}$; (c) $W_{0}=2.0 \mathrm{MW}$; between these two, the curve for $W_{0}=1.5 \mathrm{MW}$. The plasma parameters are the same as in Fig.1.

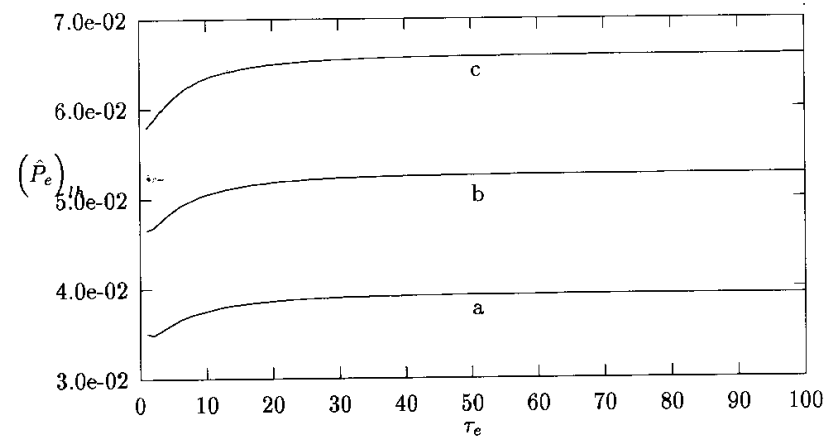

Figure 4. $\left(\hat{P}_{e}\right)_{l h}$ vs. $\tau_{e}$, at $x=0 \mathrm{~m}$, for $\omega=1.5 \omega_{l h}$, $\bar{N}_{\|}=2.53$, and $\Delta N_{\|}=0.2$. (a) $W_{0}=1.0 \mathrm{MW}$; (b) $W_{0}=1.5 \mathrm{MW}$; (c) $W_{0}=2.0 \mathrm{MW}$. The plasma parameters are the same as in Fig.1.

Regarding the results obtained with the present formulation, which uses a single wave and the corresponding diffusion coefficient [4], we observe in Fig. 5 that the parallel distribution of the ions departs from the Maxwellian form, but remains symmetrical along parallel direction. The perpendicular distribution and temperature have more significant features. In Fig. 6 and 
7 we show respectively the natural logarithm of the perpendicular ion distribution and the perpendicular ion temperature, for $W_{0}=1.0,1.5$, and $2.0 \mathrm{MW}$, at $\tau_{i}=100$. It is noticeable in both figures the effect due to the LH waves, in the diffusion along the perpendicular direction. For $W_{0}=1.5 \mathrm{MW}$ the small amount of quasilinear diffusion causes a small effect, as compared to the modification of the distribution function by the IC waves. For $W_{0}=2.0 \mathrm{MW}$, however, the diffusion due to LH waves is already very significant, and a sizable additional perpendicular flattening of the distribution is obtained.

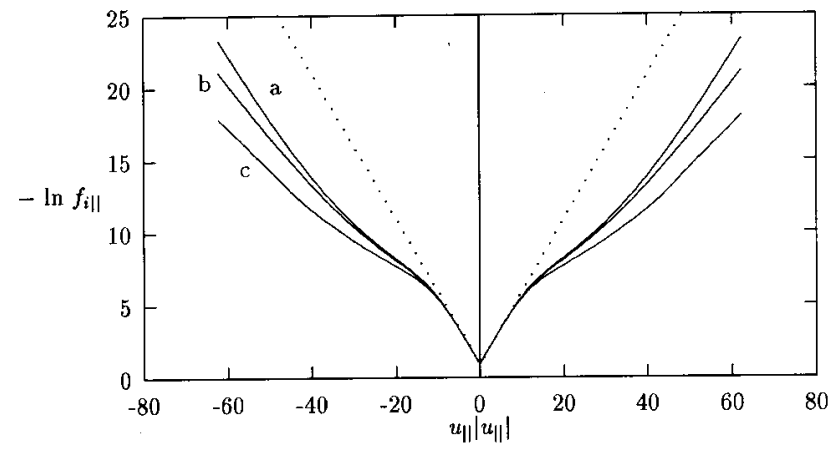

Figure 5. - In $f_{i \| \mid}$, vs. $u_{\|}\left|u_{\|}\right|$, at $x=0 \mathrm{~m}$ and $\tau_{i}=100$, for $\omega=1.5 \omega_{l h}, \bar{N}_{\|}=2.53$, and $\Delta N_{\|}=0.2$. (a) $W_{0}=1.0$ $\mathrm{MW}$; (b) $W_{0}=1.5 \mathrm{MW}$; (c) $W_{0}=2.0 \mathrm{MW}$. $D_{i c 0}=0.5$, $\bar{u}_{i c}=5.0$, and $\Delta u=1.0$. The dotted line represents the Maxwellian distribution. The plasma parameters are the same as in Fig.1.

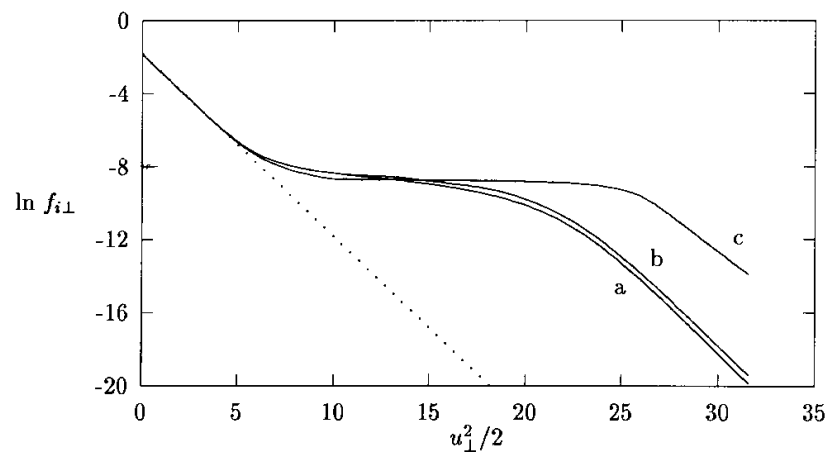

Figure 6. In $f_{i \perp}$, vs. $u_{\perp}^{2} / 2$, at $x=0 \mathrm{~m}$ and $\tau_{i}=100$, for $\omega=1.5 \omega_{l h}, \bar{N}_{\|}=2.53$, and $\Delta N_{\|}=0.2$. (a) $W_{0}=1.0$ MW; (b) $W_{0}=1.5 \mathrm{MW}$; (c) $W_{0}=2.0 \mathrm{MW}$. $D_{i c 0}=0.5$, $\bar{u}_{i c}=5.0$, and $\Delta u=1.0$. The dotted line represents the Maxwellian distribution. The plasma parameters are the same as in Fig.1.

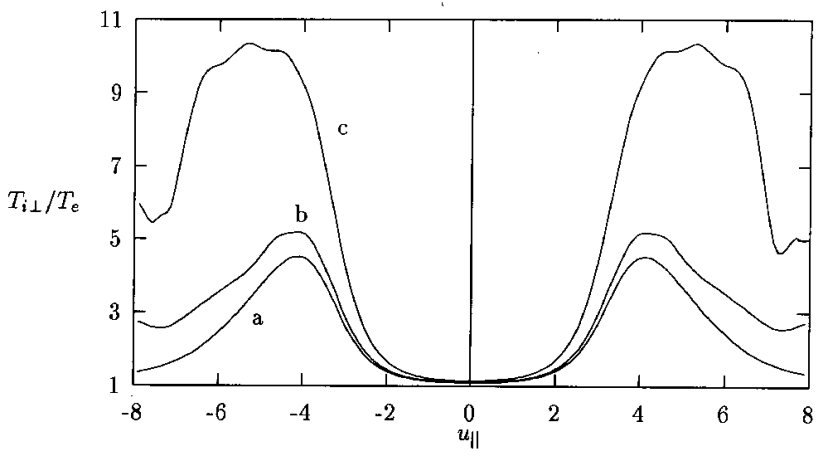

Figure 7. $T_{i} / T_{e}$ vs. $u_{\|}$, at $x=0 \mathrm{~m}$ and $\tau_{i}=100$, for $\omega=1.5 \omega_{l h}, \bar{N}_{\|}=2.53$, and $\Delta N_{\|}=0.2$. (a) $W_{0}=1.0$ $\mathrm{MW}$; (b) $W_{0}=1.5 \mathrm{MW}$; (c) $W_{0}=2.0 \mathrm{MW}$. $D_{i c 0}=0.5$, $\bar{u}_{i c}=5.0$, and $\Delta u=1.0$. The plasma parameters are the same as in Fig.1.

In Fig. 8 it is seen the time evolution of $\left(P_{i}\right)_{l h}$, for the same parameters used in Fig. 5. The comparison between the results appearing in Fig. 8 and those of Fig. 4 shows that the power dissipated by the LH waves on the ion population is null for $W_{0}=1.0 \mathrm{MW}$, is less than $10 \%$ of the power dissipated on the electron population for $W_{0}=1.5 \mathrm{MW}$, and is more than $50 \%$ of $\left(\hat{P}_{e}\right)_{l h}$ for $W_{0}=2.0 \mathrm{MW}$. It is interesting to observe that the increase of $\mathrm{LH}$ power dissipation decreases the efficiency of IC power deposition, due to the flattening of the ion distribution function caused by the LH induced diffusion. This behavior is seen in Fig. 9 , which shows the power dissipated by the model IC term, $\left(P_{i}\right)_{i c}$.

This reduction in the efficiency of IC power deposition due to high power LH waves is a non trivial behavior of the distribution function which evolves according to the quasilinear dynamics. In Fig. 10 we observe the complementary effect, by considering the time evolution of $\left(P_{i}\right)_{l h}$, for fixed LH power $\left(W_{0}=1.5 \mathrm{MW}\right)$ and four values of $D_{i c 0}$. The other parameters are as in Fig. 5. It is seen that for vanishing IC power the absence of the enhanced population of energetic ions means negligible coupling between LH waves and ions. The natural logarithm of the perpendicular ion distribution as a function of $u_{\perp}^{2} / 2$ and the perpendicular temperature as a function of $u_{\|}$are shown in Figs. 11 and 12, respectively, for $W_{0}=1.5 \mathrm{MW}$ and four values of $D_{i c 0}$. 


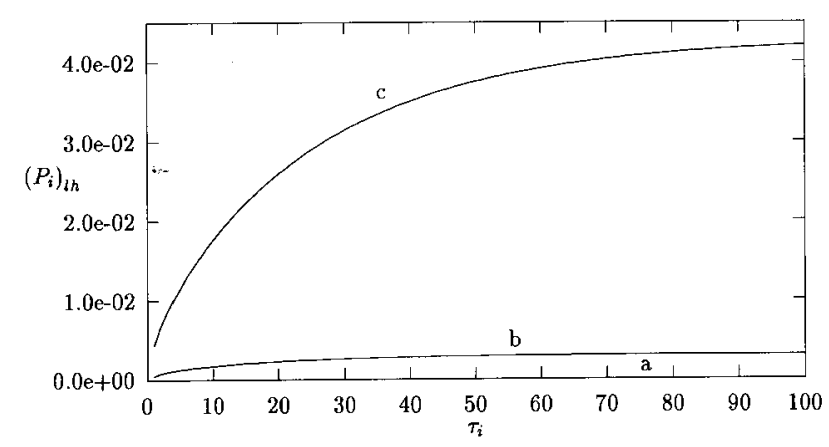

Figure 8. $\left(P_{i}\right)_{l h}$ vs. $\tau_{i}$, at $x=0 \mathrm{~m}$, for $\omega=1.5 \omega_{l h}$, $\bar{N}_{\|}=2.53$, and $\Delta N_{\|}=0.2$. (a) $W_{0}=1.0 \mathrm{MW}$; (b) $W_{0}=1.5 \mathrm{MW}$; (c) $W_{0}=2.0 \mathrm{MW}$. $D_{i c 0}=0.5, \bar{u}_{i c}=5.0$, and $\Delta u=1.0$. The plasma parameters are the same as in Fig.1.

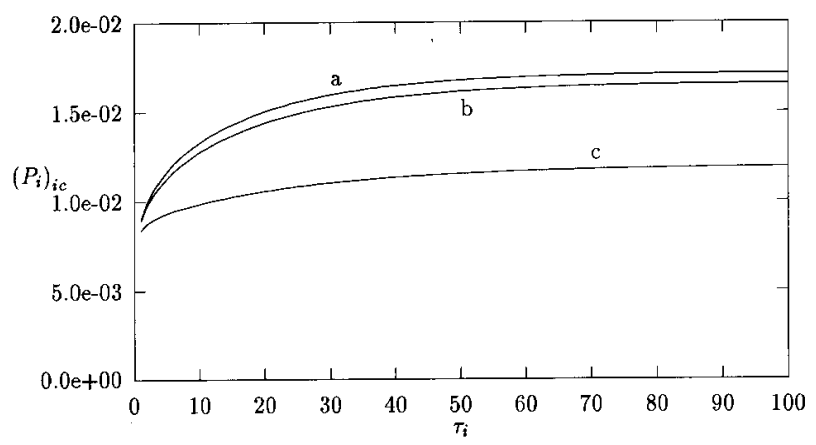

Figure 9. $\left(P_{i}\right)_{i c}$ vs. $\tau_{i}$, at $x=0 \mathrm{~m}$, for $\omega=1.5 \omega_{l h}$, $\bar{N}_{\|}=2.53$, and $\Delta N_{\|}=0.2$. (a) $W_{0}=1.0 \mathrm{MW}$; (b) $W_{0}=1.5 \mathrm{MW} ;$ (c) $W_{0}=2.0 \mathrm{MW} . D_{i c 0}=0.5, \bar{u}_{i c}=5.0$, and $\Delta u=1.0$. The plasma parameters are the same as in Fig.1.

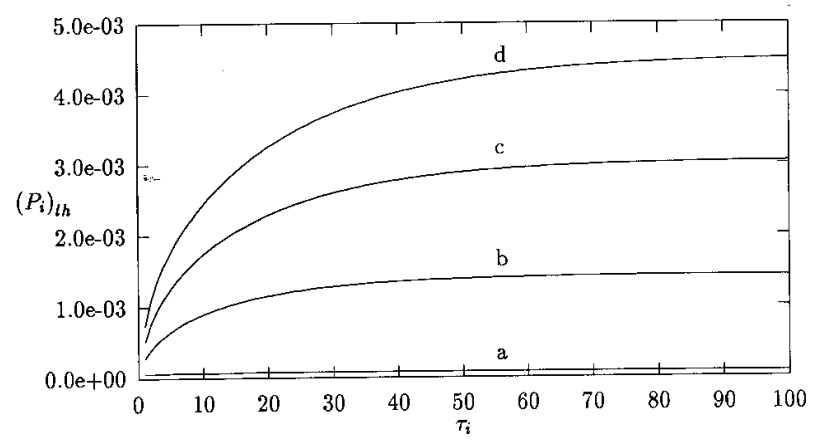

Figure 10. $\left(P_{i}\right)_{l h}$ vs. $\tau_{i}$, at $x=0 \mathrm{~m}$, for $\omega=1.5 \omega_{l h}$, $\bar{N}_{\|}=2.53, \Delta N_{\|}=0.2, W_{0}=1.5 \mathrm{MW}, \bar{u}_{i c}=5.0$, and $\Delta u=1.0$. (a) $D_{i c 0}=0.0$; (b) $D_{i c 0}=0.25$; (c) $D_{i c 0}=0.5$; (d) $D_{i c 0}=0.75$. The plasma parameters are the same as in Fig.1.

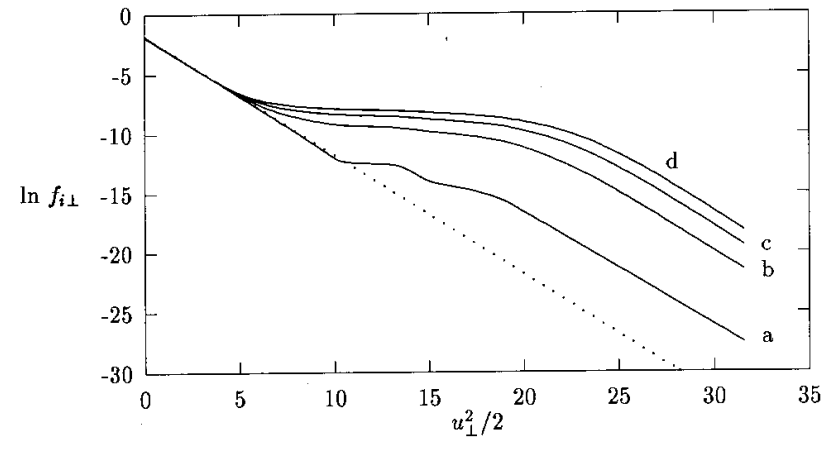

Figure 11. In $f_{i \perp}$, vs. $u_{\perp}^{2} / 2$, at $x=0 \mathrm{~m}$ and $\tau_{i}=100$, for $\omega=1.5 \omega_{l h}, \bar{N}_{\|}=2.53, \Delta N_{\|}=0.2, W_{0}=1.5 \mathrm{MW}$, $\bar{u}_{i c}=5.0$, and $\Delta u=1.0$. (a) $D_{i c 0}=0.0$; (b) $D_{i c 0}=0.25$; (c) $D_{i c 0}=0.5 ;$ (d) $D_{i c 0}=0.75$. The dotted line represents the Maxwellian distribution. The plasma parameters are the same as in Fig.1.

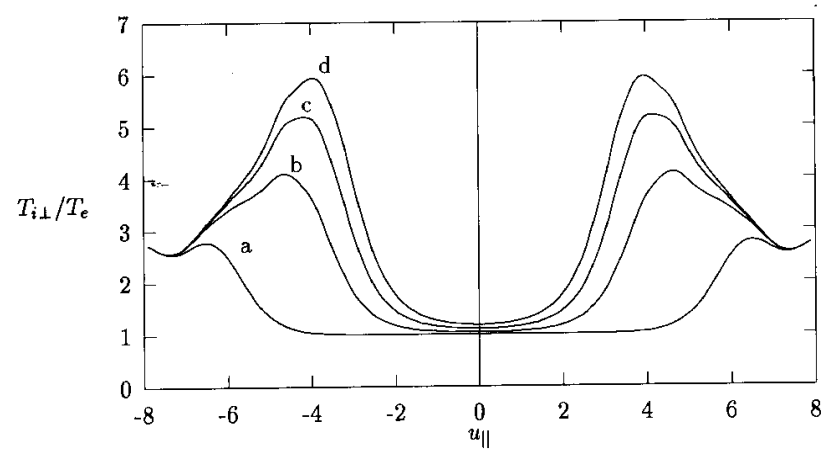

Figure 12. $T_{i \perp} / T_{e}$ vs. $u_{\|}$, at $x=0 \mathrm{~m}$ and $\tau_{i}=100$, for $\omega=1.5 \omega_{l h}, \bar{N}_{\|}=2.53, \Delta N_{\|}=0.2, W_{0}=1.5 \mathrm{MW}$, $\bar{u}_{i c}=5.0$, and $\Delta u=1.0$. (a) $D_{i c 0}=0.0$; (b) $D_{i c 0}=0.25$; (c) $D_{i c 0}=0.5$; (d) $D_{i c 0}=0.75$. The plasma parameters are the same as in Fig.1.

\section{Summary and conclusions}

In the present paper we have carried out a quasilinear analysis of the stochastic diffusion of energetic ions in a tokamak, due to the interaction with lower hybrid waves of sufficiently high intensity. The tokamak plasma and the lower hybrid wave have been described by simple models which are intended to contribute for the understanding of basic features related to the stochastic diffusion and its theoretical description.

Using quasilinear equations for the time evolution of both ion and electron distribution functions, we have considered parameters typical of present large tokamaks, The results indicate that the power dissipated by the LH waves on the ion distribution can be a significant fraction of the power dissipated on the electron 
distribution, and therefore give support to the idea that the presence of energetic ions can contribute for a redution of the efficiency of lower hybrid current drive, in qualitative agreement with experimental results. On the other hand, the energy dissipated on the ions has been shown to be null for wave powers and frequencies where it has been observed in experiments, indicating that improvements must be introduced in the theoretical description of the interaction between LH waves and energetic ions. One possible reason for the quantitative difference obtained may be the presence of a spectrum of waves instead of the single monochromatic wave assumed in the present theoretical analysis. We intend to proceed investigating the subject, and report our findings in a forthcoming publication.

\section{Acknowledgments}

This work is partially based on the contents of the dissertation submitted by L. M. Tozawa for a M.Sc. degree from the Universidade Federal do Rio Grande do Sul, and it has been partially supported by the Brazilian agencies Conselho Nacional de Desenvolvimento Científico e Tecnológico (CNPq), Financiadora de Estudos e Projetos (FINEP), and Fundação de Amparo à Pesquisa do Rio Grande do Sul (FAPERGS). Numerical computations were partially performed at the Supercomputer Center installed at the Universidade Federal do Rio Grande do Sul (CESUP-UFRGS). One of us (L. F. Z.) acknowledges useful discussions with M. C. de Andrade, I. L. Caldas, F. B. Rizzato, and R. S. Schneider.

\section{References}

[1] M. C. R. de Andrade, M. Brusati, and the JET team,
Plasma Phys. Contr. Fusion 36, 1171 (1994).

[2] M. C. R. de Andrade, PhD thesis, USP, Curso de PósGraduação em Física, 1994.

[3] E. Barbato and F. Santini, Nucl. Fusion 31, 673 (1991).

[4] C. F. F. Karney, Phys. Fluids 22, 2188 (1979).

[5] C. F. F. Karney, Phys. Fluids 21, 1584 (1978).

[6] N. J. Fisch, Phys. Rev. Lett. 41, 873 (1978).

[7] C. F. F. Karney and N. J. Fisch, Phys. Fluids 22, 1817 (1979).

[8] N. J. Fisch, Rev. Modern Phys. 59, 175 (1987).

[9] D. Anderson, V. S. Belikov, Y. I. Kolesnichenko, and M. Lisak, Phys. Fluids B 3, 3125 (1991).

[10] M. Spada, M. Bornatici, and F. Engelmann, Nucl. Fusion 31, 447 (1991).

[11] N. J. Fisch and J. Rax, Phys. Rev. Lett. 69, 612 (1992).

[12] N. J. Fisch and J. Rax, Nucl. Fusion 32, 549 (1992).

[13] J. A. Heikkinen and S. K. Sipilä, Nucl. Fusion 36, 1345 (1996).

[14] J. A. Heikkinen et al., Plasma Phys. Contr. Fusion 38, 2063 (1996).

[15] J. A. Heikkinen and T. P. Kiviniemi, Phys. Lett. A 214, 53 (1996).

[16] A. H. Kritz et al., Nuclear Fusion 18, 835 (1978).

[17] K. Ushigusa et al., Nuclear Fusion 29, 265 (1989).

[18] T. Imai et al., Nuclear Fusion 30, 161 (1990).

[19] M. Nemoto et al., Phys. Rev. Lett. 67, 70 (1991).

[20] L. F. Ziebell and L. M. Tozawa, The threshold condition for stochastic diffusion of energetic ions due to lower hybrid waves, submitted for publication (1998).

[21] I. Fidone, G. Giruzzi, G. Granata, and R. L. Meyer, Phys. Fluids 27, 2468 (1984).

[22] T. H. Stix, Nucl. Fusion 15, 737 (1975).

[23] J. D. Gaffey Jr., J. Plasma Phys. 16, 149 (1976).

[24] B. Carnahan, H. Luther, and J. Wilkes, Applied Numerical Methods (John Wiley, New York, 1969).

[25] I. Fidone et al., Nucl. Fusion 27, 579 (1987).

[26] P. R. S. Rosa and L. F. Ziebell, Plasma Phys. Contr. Fusion 34, 533 (1992). 